\section{Endocrinology of steroid hormones}

Molecular Endocrinology of the Steroid Hormones. By D. Schulster, S. Burstein and B. A. Cooke. Pp.xv+321. (Wiley: London and New York, 1976.) Cloth $£ 9.75, \$ 20$; paper $£ 4.75, \$ 10$.

THIS textbook on molecular endocrinology of the steroid hormones is an attempt to focus attention on the mechanism of action of steroid hormones. Its aim is to provide broad coverage with the minimum of fine detail in four main sections-methods, the endocrine glands, regulation of biosynthesis, and mode of action of steroid hormones.

The book is not intended to be a reference text for research workcrs, but is an account of steroid hormone biosynthesis and mode of action aimed at science students perhaps majoring in biochemistry, or at medical students. There is no doubt that a science student with a reasonable background in chemistry and biochemistry could read this text with ease.

The methodology of steroids is briefly reviewed with a fairly cursory treatment of radioimmunoassays. It might have been appropriate to have given this aspect of methodology a more extensive treatment. If the text is to be used by preclinical medical students then it is likely that most of the steroid hormone assays which they will request as practising doctors will be carried out using this method. A detailed account of the technique including its shortcomings might have been to the reader's advantage.

Biosynthesis of steroid hormones is presented in a fashion which will be acceptable to undergraduate science or medical students. Short chapters deal with the structure and function of the pituitary, hypothalamus, adrenal cortex, ovary, testes and the fetoplacental unit. These chapters each contain a brief account of the structure and ultrastructure of the endocrinc organ. its blood supply and its relationship to other tissues in the body. This section will be extremely useful to students from the physical sciences who wish to grapple with the concepts of endocrinology.

The third section of the book, which is concerned with the regulation of steroid hormone biosynthesis, contains three chapters, the largest being the control of corticoid synthesis by ACTH. Problems associated with the acceptance of the usual dogma on the mode of action of ACTH are critically reviewed in this chapter. This is followed by a brief treatment of aldosterone synthesis and a short chapter on the control of androgen and

oestrogen secretion by FSH and LH. In view of the considerable interest at the present time on the control of FSH and LH secretion, I would have preferred to have seen a more extensive treatment of the subject. For example, organic chemists and other nonbiological scientists in the pharmaceutical industry invalved in work associated with contraceptive preparations could profitably read all of this book, but this section, which would be of great interest to them, is barely seven pages in length.

The production of even a short textbook involves a considerable gestation period. The last seotion dealing with the mode of action of oestrogens, progestins and androgens covers tapics which are currently under active investigation. Most of the references contained in this section are to papers published pre-1975; because much work has emerged in 1975 and 1976, the medical or science students reading these chapters will be obliged to update the information. The basic concepts of the mode of action of steroid hormones, the localisation of the receptors and the possible nuclear events are constantly undergoing refinement and revision. These chapters are superficial, but they will whet the appetite of the

\section{Introducing prostaglandins}

Prostaglandins: An Introduction to their Biochemistry, Physiology and Pharmacology. By P. B. Curtis-Prior. Pp. xi+158. (North-Holland: Amsterdam, New York and Oxford, 1976.) Dfi. 42: \$16.25.

Tiss volume is intended as an introduction to prostaglandins for those "currently uninitiated in the subject". The author begins the main text by describing the nomenclature and biosynthesis of prostaglandins. I found the latter section rather misleading in parts-for example, the transformation of endoperoxides to prostaglandins is depicted (wrongly) as a reversible reaction. Sections covering prostaglandin catabolism, inhibitors of biosynthesis and reccptor antagonists, are followed by a chapter entitled "Prostaglandinlike substances" (mainly polypeptides). which I felt did not really belong in the book at all. The remainder of the text largely comprises an account of the effects of prostaglandins in physiological and pathological processes. although little was said about their role in the control of parturition or platelet aggregation-surely two areas where the case for prostaglandin involvement is stronger than most? The student; and the enthusiast will go on to read beyond this text.

It is regrettable that the mode of action of aldosterone does not receive a more detailed treatment. This is one of the few examples in steroid endocrinology where the investigator can in fact obtain a fairly precise physiological indicator of the action of the hormone in an in vitro system:

This treatment of the endocrinology of steroid hormones will be welcomed by many teachers. The texthook will be valuable to students who are reading biochemistry either for a general degree or for an honours degree, and many of the problems associated with the interpretation of the mode of action of steroid hormones are highlighted. Since the subject is so wide the treatment is superficial in parts; but it is always interesting. readable and relatively free from errors. The book can be recommended as a straightforward, clear account of the concepts of molecular endocrinology of steroid harmones in the mid-1970s.

G. S. Boyd

G. S. Boyd is Chairman of the Department of Biochemistry at the University of Edinburgh. UK, and Honorary Director of an $M R C$ group on sterol metabolism.

final chapter deals rather perfunctorily with the important topic of biosynthesis of thromboxanes and other non-prostaglandin products of arachidonate.

The text is produced by a process of direct photographic reproduction of the typcscript, and the difficulties this causes in the arrangement of the figures give this (otherwise well produced book) a slightly unbalanced look. I found the quality of the artwork rather variable, mainly because half of the figures are reproduced from other sources: a practice which leads to trouble in the final chapter where the wrong structure of thromboxane A. is given.

This book is easy to read. but I found the chapters rather superficial and had the impression several times that the author was a little out of touch with the mainstream of prostaglandin research. There is, however, no "primer" on prostaglandins to which the "uninitiated" can refer, and no doubt many will be glad to find this information collected in a basic text. The book contains adequate references for further study. $\quad$ R. J. Flower

R. J. Flower is a research pharmacologist within the Wellcome Foundation. Beckenham. Kent, UK. 